\title{
Revista Colombiana de

\section{Capítulo 4. Planificación de las clínicas de falla cardiaca, objetivos, infraestructura y personal}

\section{Gina González}

\author{
Medicina Interna y Cardiología, Fundación Santa Fe de Bogotá, Bogotá, Colombia
}

Recibido el 16 de noviembre de 2015; aceptado el 12 de enero de 2016

Disponible en Internet el 1 de febrero de 2016

\section{PALABRAS CLAVE Insuficiencia cardiaca; Pronóstico; Estudios de seguimiento}

\section{KEYWORDS}

Heart failure;

Prognosis;

Follow-up studies

\begin{abstract}
Resumen
Introducción: Los modelos de atención especializados en insuficiencia cardiaca, emergen como una estrategia para mejorar el pronóstico de estos pacientes.

Objetivo: Describir los elementos necesarios para el funcionamiento de las clínicas de falla cardiaca.

Metodología: Revisión de la literatura disponible.

Conclusión: El programa de insuficiencia cardiaca debe adaptarse a las características geográficas y logísticas de las que dispone cada área sanitaria, con componentes básicos como un cardiólogo y una enfermera, especializados en insuficiencia cardiaca, así como un coordinador asistencial que permita el enlace con los diferentes niveles de atención del sistema de salud. (c) 2016 Publicado por Elsevier España, S.L.U. en nombre de Sociedad Colombiana de Cardiología y Cirugía Cardiovascular. Este es un artículo Open Access bajo la licencia CC BY-NC-ND (http://creativecommons.org/licenses/by-nc-nd/4.0/).
\end{abstract}

Chapter 4. Heart failure clinic planning, objectives, infrastructure and personnel

\section{Abstract}

Introduction: Specialist heart failure care models are emerging as a strategy to improve the prognosis of these patients.

Objective: To describe the elements required to operate heart failure clinics.

Methodology: A review of the available literature.

Conclusion: A heart failure programme should adapt to the geographical and logistical features of each health area, with basic components such as a cardiologist and a nurse, who are 
specialists in heart failure, and a healthcare coordinator to provide a link with the different care levels of the healthcare system.

(c) 2016 Published by Elsevier España, S.L.U. on behalf of Sociedad Colombiana de Cardiología y Cirugía Cardiovascular. This is an open access article under the CC BY-NC-ND license (http://creativecommons.org/licenses/by-nc-nd/4.0/).

\section{Introducción}

Los modelos de atención especializados en insuficiencia cardiaca surgieron como una estrategia para mejorar el pronóstico de los pacientes ${ }^{1}$. Existen dos componentes que pueden ser responsables de estos resultados: la optimización terapéutica tras una mejor aplicación de la evidencia científica y la interrelación entre factores médicos, psicosociales y ambientales que rodean a los pacientes con insuficiencia cardiaca y sus cuidadores. En el tratamiento de otras enfermedades crónicas ya están consolidados muchos equipos multidisciplinares, tales como los grupos de diabetes mellitus, geriatría y oncología, que ofrecen cuidados en hospitales de día y unidades de tratamiento y asistencia rápida. No existe un modelo único de atención en insuficiencia cardiaca. De ahí que el programa de insuficiencia cardiaca deba adaptarse a las características geográficas y logísticas de las que dispone cada área sanitaria, intentando incluir componentes básicos como un cardiólogo y una enfermera, especializados en insuficiencia cardiaca, así como un coordinador asistencial que permita la conexión con los diferentes niveles de atención.

En 2006 Lupón et al. propusieron la siguiente clasificación en los modelos de atención:

1. Según los miembros que la componen: enfermeros, equipo médico, fisioterapeutas, nutricionistas, psicólogos, etc.

2. Según su localización física y tipo de pacientes bien sea atención hospitalaria u hospital de día, atención extrahospitalaria (centros de atención primaria) o asistencia domiciliaria.

3. Según el tipo de intervención, ya sea educativa y soporte a cuidadores o una intervención médica y educativa integral farmacológica y no farmacológica.

Estudios publicados han demostrado que los pacientes atendidos en programas liderados por cardiólogos ${ }^{2,3}$, tienen mejor pronóstico y mayor supervivencia en comparación con los que son vistos sólo por médicos de familia ${ }^{4}$.

La planificación de la unidad de insuficiencia cardiaca comienza con el diagnóstico de las necesidades del entorno sanitario y el empoderamiento de un líder clínico motivado y capaz de dirigir actuaciones; posteriormente es preciso realizar la evaluación y la adaptación de los recursos para empezar el proyecto.

Las características esenciales son las siguientes:

1. Enfoque multidisciplinar (conformado por cardiólogos, médicos de atención primaria, enfermeras, farmacéuti$\cos$, nutricionistas, fisioterapeutas, etc.).
2. Selección de pacientes: el programa debe estar dirigido a pacientes sintomáticos con insuficiencia cardiaca con fracción de eyección reducida o preservada, clasificados como pacientes de alto riesgo.

3. Grupo de trabajo competente con formación específica en la atención de pacientes con insuficiencia cardiaca.

\section{Dentro de los componentes se destacan:}

1. Proveer y monitorizar el tratamiento médico óptimo y el manejo de dispositivos.

2. Educar al paciente con énfasis en el autocuidado y el cumplimiento terapéutico, el entrenamiento sobre los síntomas y signos de alarma y el régimen flexible de diuréticos.

3. Planear y ejecutar el seguimiento estructurado desde el alta hospitalaria (capítulo 6).

4. Hacer seguimiento presencial, telefónico, a domicilio o incluso el seguimiento remoto por telemedicina (capítulo 13).

5. Estar en capacidad de proveer un acceso fácil al equipo multidisciplinar sanitario de modo que haya ingreso oportuno a las visitas, respuesta proactiva a signos de alarma, atención precoz a la descompensación y tratamiento integral, para lo cual es imprescindible contar con el concepto de un hospital de día (capítulo 3) o un centro de tratamiento ambulatorio especializado en insuficiencia cardiaca.

6. Ofrecer soporte psicosocial al paciente, los familiares y los cuidadores de acuerdo con los diferentes estadios de la enfermedad, facilitando diferentes vías clínicas en función de las necesidades del paciente con opciones específicas y avanzadas de tratamiento basadas en la evidencia ${ }^{5}$.

\section{¿Cómo estructurar los procesos?}

El modelo de gestión en insuficiencia cardiaca contempla la implementación de vías clínicas, las cuales son un conjunto de intervenciones planteadas para una población específica, desarrolladas en un tiempo determinado, en búsqueda de la eficiencia y la coordinación del proceso asistencial. Básicamente, permiten estandarizar la atención clínica, a través de la reducción de la variabilidad en las intervenciones y el aseguramiento del cumplimento de metas definidas para un grupo de pacientes cuya enfermedad tiene un curso clínico predecible. Para que las intervenciones realizadas tengan valor dentro del ámbito de la gestión clínica deben guiarse en la práctica basada en la evidencia. 
La implantación de la gestión por procesos tiene tres fases:

1. Fase estratégica: en esta se plantean los objetivos globales del proyecto, se busca la participación de líderes asistenciales y de política sanitaria, y se establecen los valores, la misión y objetivos estratégicos. Igualmente, se estructuran los mapas de procesos y guías de diseño de proceso.

2. Fase de desarrollo: aquí se identifican los líderes encargados de coordinar, se elige el equipo colaborador integral y multidisciplinario, se realiza la orientación hacia resultados y se definen los sistemas de medición, monitorización y evaluación de resultados. Con la elaboración de los indicadores, termina la fase de desarrollo e inicia la de implantación. Es necesario esbozar el registro clínico en una base de datos para realizar una primera caracterización de la población con insuficiencia cardiaca.

3. Fase de implantación: en esta se difunden los valores y los objetivos y se adecúan las competencias de los profesionales, con énfasis en la implicación de los mismos. De igual forma se realiza el proceso de formación de otros de ellos.

\section{¿Cómo empezar?}

Al realizar una valoración de nuestro entorno o situación, se analizan las posibilidades reales de colaboración con los servicios de apoyo como Medicina Interna, Medicina Familiar, Geriatría, Psicología, Psiquiatría, Fisiatría, Fisioterapia, etc. Se debe buscar y promover entre diferentes servicios de apoyo el beneficio mutuo, el planteamiento de objetivos, la realización de protocolos factibles y la formulación de trabajos de investigación.

\section{Necesidades físicas}

De acuerdo con la infraestructura disponible se hace una adaptación para optimizar los recursos disponibles. En un modelo enfocado en la atención de pacientes hospitalarios en hospitales de mediana y alta complejidad, se requerirá un área de hospitalización conforme a las normas de habilitación de la legislación colombiana y un área de atención ambulatoria a pacientes, en la que, como mínimo, se disponga de un consultorio para atención médica dotado con camilla de exploración, tensiómetro, báscula, cinta métrica, fonendoscopio, oxímetro de pulso, computador de escritorio con acceso a Internet y teléfono. Para favorecer la identidad de la unidad, es importante contar con una extensión de teléfono propia, correo electrónico e idealmente un espacio físico tipo oficina que le permita a los pacientes tener un sitio de referencia diferente a la consulta, por ejemplo para fines administrativos como recepción e intercambio de documentos, formularios de no Plan obligatorio de salud (POS), autorizaciones, etc., que son importantes en este sistema de salud.

La enfermera entrenada en insuficiencia cardiaca debe portar un teléfono móvil por medio del cual realizará el seguimiento telefónico y atenderá llamadas de pacientes en los horarios que se definan de acuerdo con la disponibilidad de la coordinación del procedimiento (es muy importante definir estas horas y dejarlas claras a pacientes y cuidadores).

El hospital de día (capítulo 3) es un área que desempeña un papel relevante en el proceso de atención continuada a pacientes con insuficiencia cardiaca. Se trata de un espacio de atención ambulatoria, especializado para pacientes de alto riesgo en el cual, además de realizar las actividades de educación, titulación de fármacos y consulta médica en insuficiencia cardiaca, se pueden administrar tratamientos complementarios como diuréticos endovenosos, hierro parenteral o terapias del final de vida en pacientes con insuficiencia cardiaca (incluyendo uso de inotrópicos intermitentes con intención paliativa). Este espacio debe estar provisto de sillones cómodos para la administración de medicamentos, insumos para accesos venosos periféricos, bombas de infusión para administración de medicamentos especiales y elementos para toma y transporte de muestras, así como conexión con farmacia o disponibilidad de un estante de medicamentos básicos (como furosemida en ampollas, viales de hierro parenteral, hidroclorotiazida en comprimidos orales, suplementos de potasio oral). Debe disponerse de un electrocardiógrafo y un carro de paro cardiaco con desfibrilador externo. Se necesita una fuente de oxígeno, aire y succión de pared, así como elementos para administrar oxígeno humidificado. Se requiere, así mismo, de una silla de ruedas, camilla de transporte y un circuito definido para el traslado de pacientes al servicio de urgencias en caso de requerirlo, lo que implica tener personal de apoyo dentro de este circuito.

Los materiales educativos audiovisuales complementan acertadamente el proceso educativo a pacientes y cuidadores tanto en hospitalización como en servicio ambulatorio, y deben estar disponibles para reforzar los procesos educativos de los pacientes ${ }^{6}$. Estos pueden ser generados de manera conceptual y gráfica por el mismo equipo multidisciplinario. Otra opción es utilizar el material validado por sociedades científicas como la europea de cardiología por medio de su página web www.heartfailurematters.org, disponible en varios idiomas.

\section{Necesidades de personal}

Como se ha mencionado, existen múltiples definiciones del carácter multidisciplinario, pero de acuerdo con la literatura disponible ${ }^{7}$, el modelo ideal para el desarrollo de una clínica de falla es aquel centralizado en cardiología. El rol del cardiólogo se destaca por el liderazgo en gestión y la asistencia tanto en hospitalización como en el entorno ambulatorio, la aplicación intensiva de las recomendaciones clínicas basadas en la evidencia, la formación del personal no médico sanitario, la implementación de guías, coordinación general de las funciones de los todos los colaboradores, la investigación y el control de calidad, la monitorización de efectos adversos de las terapias, la selección de pacientes candidatos a trasplante cardiaco, la elegibilidad de terapias de alta tecnología en insuficiencia cardiaca y la derivación de los pacientes a un centro de mayor o menor complejidad. Los médicos de familia y los médicos generales de atención primaria son los encargados de dar continuidad a los cuidados del paciente y su prescripción de acuerdo con 
las directrices de la unidad. Los médicos generales de la unidad tienen dentro de sus funciones, extender la cobertura horaria de atención, superponerse horariamente con el cardiólogo y la enfermera haciendo especial énfasis en el control y seguimiento clínico de pacientes con insuficiencia cardiaca y otras comorbilidades propias del ámbito generalista y de la cardio-geriatría, el soporte y la validación de la titulación de fármacos realizados en conjunto con la enfermera, así como el reclutamiento, seguimiento y registro de pacientes como el que realizan el cardiólogo y la enfermera especializada ${ }^{8}$.

La enfermera es el principal ejecutor de las actividades asistenciales con los pacientes, lo que incluye intervención educativa intrahospitalaria, fortalecimiento del autocuidado 9 , diagnóstico social del enfermo, evaluación de la competencia del cuidador, evaluación de agotamiento del cuidador e integración de las actividades con enfermería en atención primaria y servicios domiciliarios. Atiende en el Hospital de día, realiza visita conjunta, es responsable de la mayoría de la educación sanitaria en insuficiencia cardiaca ambulatoria programada, ejecuta el protocolo de seguimiento telefónico, es la veedora de la evaluación global del funcionamiento de la unidad y participa activamente en la evaluación clínica del paciente y la titulación de fármacos con impacto pronóstico en insuficiencia cardiaca ${ }^{10}$. Cada hospital de agudos debe tener una enfermera especializada en insuficiencia cardiaca por cada 100000 habitantes $^{7}$, objetivo propuesto por la Sociedad europea de cardiología.

\begin{tabular}{ll}
\hline Profesional & Funciones \\
\hline Cardiología & $\begin{array}{l}\text { Estudio de la cardiopatia, la titulación de } \\
\text { medicación, la educación, el diagnóstico y } \\
\text { el manejo temprano de } \\
\text { descompensaciones, entre otras terapias. }\end{array}$ \\
Enfermería & $\begin{array}{l}\text { Evaluación social, educación, autocuidado, } \\
\text { vacunación, seguimiento telefónico, } \\
\text { manejo de Hospital de día, coordinación }\end{array}$ \\
& $\begin{array}{l}\text { Evaluación, dieta, restricción hídrica, } \\
\text { ajuste a comorbilidades; taller de manejo } \\
\text { de líquidos y dieta }\end{array}$ \\
& $\begin{array}{l}\text { Diagnóstico piscosocial, apoyo al paciente y } \\
\text { a la familia, direccionamiento a Psiquiatría; }\end{array}$ \\
& $\begin{array}{l}\text { Taller de manejo del estrés y taller de } \\
\text { cuidadores } \\
\text { Régimen de ejercicio para paciente con } \\
\text { Rehabilitación }\end{array}$ \\
& $\begin{array}{l}\text { insuficiencia cardiaca, clase funcional } \\
\text { mayor o igual a Il }\end{array}$ \\
\hline
\end{tabular}

\section{Parámetros de calidad y plan funcional}

En este documento se consigna de forma minuciosa todo el proyecto, el cual debe contener los ítems necesarios para convencer al gerente, director médico y jefe de servicio, a fin de definir de forma concreta y organizada las interacciones de los profesionales implicados para involucrarlos como protagonistas o colaboradores. Un apartado muy robusto es la justificación de la creación del programa de insuficiencia cardiaca. Aquí se debe describir la importancia de la pandemia de insuficiencia cardiaca con datos epidemiológicos, morbilidad, mortalidad, costo social, además de las cargas directas e indirectas a nivel económico derivadas de la enfermedad y sus consecuencias. Debe realizarse un análisis del propio entorno con datos de ingresos por insuficiencia cardiaca en el hospital, datos de reingresos, número de consultas externas, número de consultas a urgencias, características de los pacientes, días de estancia media, recursos utilizados y costos, al igual que presentar estos datos en forma de gráfica con la tendencia en función del tiempo. Podría ser importante discriminar de acuerdo con los servicios clínicos a cargo (Cardiología, Medicina Interna, Geriatría).

En el plan funcional se debe describir la estructura actual y el protocolo de trabajo que maneja el hospital definiendo cargas de trabajo, áreas de hospitalización convencional, consultas externas, análisis de las cargas de trabajo y de los efectos de la intervención, limitaciones actuales de la estructura, descripción de recursos físicos y humanos, así como de objetivos generales, asistenciales, docentes y de investigación. Dentro de los anexos a esta sección, se incluye el organigrama, el protocolo de la futura unidad de insuficiencia cardiaca, los criterios de inclusión de pacientes, la explicación de qué intervención se hará y dónde, la descripción de protocolos específicos, la previsión volumen de actividad y los recursos necesarios para llevar a cabo los protocolos. Para cuantificar los resultados de la intervención se deben usar los indicadores, los cuales pueden ser de proceso y de resultado. Los primeros pueden ser cuantitativos, los cuales reflejan actividades que se repiten constantemente (demoras en derivación, tiempos de respuesta, días ingreso, calidad de vida). Los segundos evalúan aspectos de cumplimiento de protocolos y control de equipos. También hay indicadores de calidad del proceso y de los resultados. Los indicadores de resultado son de efectividad, de satisfacción y de eficiencia (tasa de reingreso, tasa de frecuentación a urgencias, número de primeros ingresos, reingresos y reingresos múltiples, determinación de la mortalidad de los pacientes con insuficiencia cardiaca y número de pruebas complementarias, como algunos ejemplos de este tipo de indicadores ${ }^{11}$. Un anexo final es el curriculum vitae de las personas que lideran el proyecto, destacando sus competencias específicas en insuficiencia cardiaca.

\section{Conclusiones}

La planificación adecuada de una clínica de falla cardiaca incluirá los siguientes elementos:

- Identificación de las necesidades del entorno: definir objetivos del proyecto como reducir los ingresos hospitalarios por insuficiencia cardiaca, mejorar la clase funcional de los pacientes, brindar atención de calidad y seguir los lineamientos de las guías.

- Personal necesario: los programas deben ser multidisciplinarios, contar con un cardiólogo y una enfermera con entrenamiento específico en el cuidado de pacientes con insuficiencia cardiaca y adicionalmente recibir el apoyo de Psicología, Rehabilitación cardiaca, Nutrición y Servicio farmaceútico.

- Espacio físico: es importante contar con un espacio dedicado a la atención de los pacientes. 
- Vías clínicas y protocolos: permiten brindar atención estandarizada y de calidad.

- Integración con niveles de menor complejidad.

- Indicadores que faciliten el seguimiento del proceso.

\section{Conflicto de intereses}

Los autores declaran no tener ningún conflicto de intereses.

\section{Bibliografía}

1. Jessup M, Brozena S. Heart failure. N Engl J Med. 2003;348: 2007-18.

2. Indridason OS, Coffman CJ, Oddone EZ. Is specialty care associated with improved survival of patients with congestive heart failure? Am Heart J. 2003;145:300-9.

3. Ansari M, Alexander M, Tutar A, Bello D, Massie BM. Cardiology participation improves outcomes in patients with new-onset heart failure in the outpatient setting. J Am Coll Cardiol. 2003;41:62-8.

4. Ezekowitz JA, van WC, McAlister FA, Armstrong PW, Kaul P. Impact of specialist follow-up in outpatients with congestive heart failure. CMAJ. 2005;172:189-94.

5. McMurray JJ, Adamopoulos S, Anker SD, Auricchio A, Böhm M, Dickstein K, et al. Task force for the diagnosis and treatment of acute and chronic heart failure 2012 of the European Society of Cardiology. Developed in collaboration with the Heart Failure Association (HFA) of the ESC. Eur J Heart Fail. 2012;14: 803-69.

6. McAlister FA, Stewart S, Ferrua S, McMurray JJ. Multidisciplinary strategies for the management of heart failure patients at high risk for admission: a systematic review of randomized trials. J Am Coll Cardiol. 2004;44:810-9.

7. McDonagh T, Blue L, Clark A, Dahlstro "MU, Ekman I, Lainscak M, et al. European Society of Cardiology Heart Failure Association Standards for delivering heart failure care. Eur J Heart Fail. 2011;13:235-41.

8. Philbin EF, Weil HF, Erb TA, Jenkins PL. Cardiology or primary care for heart failure in the community setting: process of care and clinical outcomes. Chest. 1999;116:346-54.

9. Lainscak M, Blue L, Clark AL, Dahlström U, Dickstein K, Ekman I, et al. Self-care management of heart failure: practical recommendations from the Patient Care Committee of the Heart Failure Association of the European Society of Cardiology. Eur J Heart Fail. 2011;13:115-26.

10. Mariani M, Vella G, Bianchi C, Verde S, De MR, Pirelli S. Implementation of beta-blockade in elderly heart failure patients: role of the nurse specialist. Eur J Cardiovasc Nurs. 2008;7:196-203.

11. Gustafsson F, Arnold JM. Heart failure clinics and outpatient management: review of the evidence and call for quality assurance. Eur Heart J. 2004;25:1596-604. 